\title{
Hacer negocios con adolescentes: una experiencia práctica de $\mathrm{EFE}^{1}$
}

\author{
ALEJANDRA HERNÁNDEZ RUIZ ${ }^{2}$ \\ Universidad de Valencia \\ alejandra.hernandez@uv.es
}

\begin{abstract}
Resumen: La presente propuesta nace a partir de la implantación de la Ley Educativa italiana Alternanza Scuola-Lavoro, que obliga a los estudiantes de entre 15 y 19 años a realizar horas lectivas sobre el mundo de los negocios en una lengua extranjera. Para dar respuesta a esta demanda, se diseña un curso de Español con Fines Específicos (EFE) dirigido a adolescentes. Esta experiencia práctica pretende comprobar si los contenidos y los materiales (1) resultan útiles para los jóvenes en su aprendizaje, (2) son válidos para la consecución de sus objetivos y (3) logran aumentar su motivación. Para ello, tras el diseño del programa y su puesta en práctica, se elaboran unos Cuestionarios de (Auto)Evaluación, los cuales conforman el punto central de la propuesta, pues permitirán evidenciar si nuestros propósitos han sido alcanzados. Los resultados de estos cuestionarios muestran la satisfacción con respecto al curso por parte de los estudiantes, así como la consecución de todos los planteamientos iniciales.
\end{abstract}

Palabras clave: Español con Fines Específicos, Español de los Negocios, adolescentes, Cuestionarios de (Auto)Evaluación, $A S L$

\section{Doing business with teenagers: a SSP practical experience.}

Abstract: The present proposal was born from the implementation of the Italian Educational Law Alternanza Scuola-Lavoro, which requires students between 15 and 19 years old to carry out teaching hours about Business in a foreign language. To respond to this demand, a Spanish for Specific Purposes course (SSP) for adolescents is designed. This practical experience aims to verify if the contents and materials (1) are useful for students and they can extract a significant learning, (2) are valid to achieve the purposed goals and (3) they are able to increase their motivation. For this, after the program design and its application in class, some (Self-)Assessment Questionnaires which form the central point of the proposal- are elaborated, since they will allow to show if our purposes and goals have been reached. The results of the tests show the students' satisfaction respect to the course, as well as the achievement of the initial objectives.

Key words: Spanish for Specific Purposes, Spanish for Business, adolescents, (Self)Assessment Questionnaires, $A S L$

1 Este artículo ha sido posible gracias al proyecto de investigación FFI2016-77842-P, Unidades discursivas para una descripción sistemática de los marcadores del discurso en español (UDEMADIS), financiado por el MINECO, la AEI y los fondos FEDER.

2 La autora del presente trabajo es beneficiaria de la ayuda para la formación de personal investigador de carácter predoctoral en el marco del subprogama Val+ID de la Conselleria de Educació, financiado por la Generalitat Valenciana y el Fondo Social Europeo. 


\section{Introducción}

\section{1. ¿Por qué un curso de EFE para adolescentes?}

La idea de diseñar un programa de EFE para adolescentes nace por la implantación de la ley italiana Alternanza Scuola-Lavoro ${ }^{3}$ (en adelante $A S L$ ), en la que estudiantes con edades comprendidas entre los 15 y los 19 años visitan España para realizar un curso teórico-práctico sobre el mundo de los negocios.

Así, desde la academia AIP Idiomas ${ }^{4}$ se elabora una programación enfocada a cubrir los objetivos planteados en la $A S L$, ya que, tras la revisión de manuales (tanto de EFE como de EG, Español General ${ }^{5}$ ), se apreció la falta de adecuación de estos al público al que pretendíamos dirigirlo, pues no se ajustaban a las demandas de la ley italiana, así como tampoco lo hacían a la edad de los alumnos, pues los materiales de español de los negocios suelen estar enfocados a adultos que, o bien forman parte del mercado y del mundo laboral, o bien están en contacto con él.

\subsection{Objetivos}

Puesto que nos encontramos ante una nueva planificación creada ad hoc para cubrir unas necesidades específicas, su validez y eficacia deben ser testadas: si dicha propuesta pretende aportar una nueva visión de EFE, dirigida a un nuevo público, se hace necesaria una evaluación llevada a cabo por el propio grupo meta ${ }^{6}$.

Por tanto, esta experiencia práctica tiene como principal objetivo comprobar si los contenidos y los materiales del curso de Español de los Negocios para adolescentes:

a. resultan útiles para los estudiantes y extraen un aprendizaje significativo de ellos, así como si se adaptan a las necesidades concretas del grupo meta.

b. son válidos para la consecución de sus objetivos y los de $A S L$.

c. aumentan su motivación e interés en el Español de los Negocios.

Para ello, se elaboran un Cuestionario de Evaluación del curso y uno de Autoevaluación de sus conocimientos y habilidades. Estos conforman la parte fundamental de este

\footnotetext{
${ }^{3}$ La implantación de la Ley Educativa italiana Alternanza Scuola-Lavoro consiste en la realización de entre 200 y 400 horas lectivas -tanto teóricas como prácticas-por parte de estudiantes de escuela media y superior (edades comprendidas entre los 15 y los 19 años) sobre el mundo de los negocios. El objetivo de este nuevo plan de estudios es facilitar su inserción en el mercado laboral, con una mayor formación teórica a la par que práctica. Además, estas horas han de ser realizadas en una lengua extranjera, a elección de sus respectivos centros, lo cual les permite entrar en contacto con el mercado comercial foráneo. Por otra parte, pueden cursarlas bien en su centro, bien en otro país en situación de inmersión lingüística, como es nuestro caso. Pueden consultarse más detalles en https://labuonascuola.gov.it/

${ }^{4}$ AIP Idiomas: Advisers for International Programs in Spain es el nombre completo de la academia.

${ }^{5}$ Los manuales revisados, entre otros, son: Al día (Ed. Sgel), Socios y Expertos (Ed. Difusión) y Empresa siglo XXI (Ed. Edinumen). No obstante, aunque el uso exhaustivo de estos se descarta por la falta de adecuación al contexto de uso, sí que se toman de ellos algunas actividades y textos para el nuevo programa: en lugar de seguir únicamente un manual, se crea una planificación nueva a partir de materiales extraídos de todos estos, entre otros documentos tomados de fuentes diversas.

${ }^{6}$ Nuestro grupo meta serán estudiantes italianos, en edad adolescente, y que posean un nivel intermedio de español (B1-B2: se exige que tengan unos conocimientos medianamente amplios a nivel léxico y gramatical). Concretamente, el grupo meta con el que se lleva a cabo este estudio está formado por 28 alumnos de 16 y 17 años que se sitúan en un nivel B1+/B2.
} 
trabajo, pues permitirán evidenciar si nuestros propósitos han sido alcanzados y saber si el diseño curricular es adecuado para los grupos meta. En definitiva, si la experiencia práctica tiene éxito y es percibida por los alumnos como rentable y significativa para su vida académico-laboral.

\section{Marco teórico}

\subsection{La Sociedad de la Información y la Comunicación (SIC)}

Las civilizaciones avanzan, evolucionan y, con ellas, sus habitantes, la cultura intrínseca a cada grupo social y la lengua. Así pues, tras la Segunda Guerra Mundial, comenzaron a sucederse una serie de cambios que quedaron asentados en la sociedad. La compleja situación socioeconómica, política y educativa de la segunda mitad del siglo XX y principios del XXI (consolidación de nuevas tendencias políticas, económicas y tecnológicas: movimientos migratorios, integración de mercados e internacionalización económica, globalización de la información o empleo de las Tecnologías de la Información y la Comunicación -TIC-, entre otras) ha tenido influencia en cada una de las actividades humanas cotidianas y ha demostrado la gran importancia que ha adquirido el factor información-conocimiento para el desarrollo científico y económico. Ante esta nueva sociedad, se ha hecho manifiesta también la necesidad de una formación más profunda y especializada en lenguas extranjeras, que permita el acceso a todo tipo de información, "con el fin de alcanzar la competencia comunicativa en ámbitos académicos y profesionales» (Aguirre Beltrán, Sánchez Lobato y Santos Gargallo 2012: 275) y que persigue un objetivo todavía mayor:

Establecer relaciones diplomáticas, financieras, laborales y comerciales con todos los pueblos de la Tierra; esto es, para desenvolverse efectiva y eficazmente en contextos que precisan unas destrezas y unas estrategias de comunicación determinadas y unos conocimientos adecuados a las convenciones de uso en estos contextos.

Este nuevo modelo social que se ha gestado durante finales del siglo XX y principios del XXI ha demostrado que, si se pretende un desarrollo económico, el tándem informaciónconocimiento es la base para alcanzarlo (Aguirre Beltrán 2000). Así pues, los factores citados anteriormente -ligados todos ellos a la necesidad de acceder de forma rápida y concisa a la información, de movernos globalmente y de comunicarnos- han impactado de manera directa y positiva en la enseñanza y aprendizaje de lenguas, lo cual ha generado un aumento de la demanda de lenguas extranjeras como materia de estudio (Grau Mena 2009).

Y no solo el cambio que ha sufrido la sociedad influye en el crecimiento de la enseñanza de lenguas extranjeras, sino también el vínculo que une a la lengua con las transacciones comerciales: «las lenguas son un factor de transmisión de conocimientos y de penetración comercial para todo tipo de productos y servicios, teniendo en cuenta que el comercio es básicamente comunicación y la lengua es el instrumento de esa comunicación» (Aguirre Beltrán, Sánchez Lobato y Santos Gargallo 2012: 276). Por otra parte, se suma el estrecho lazo entre lengua y cultura: «la lengua — su conocimiento - nos ayuda a ser «invitado» a participar en la sociedad y, por ende, a conocer sus formas culturales de intercomunicación» (276). 


\subsection{El español como lengua con fines específicos}

Como indica Aguirre, ante una situación socio-económica de tal calibre, el dominio de otras lenguas es fundamental porque gracias a ellas somos capaces de comunicarnos: «el lenguaje es el instrumento que facilita y acelera el acceso a la información y permite la comunicación del conocimiento y del conocimiento consolidado, el saber» (2000: 34).

La orientación hacia un planteamiento más comunicativo del estudio de lenguas debido a las necesidades originadas para transmitir información y conectarnos con el resto del mundo- generó, en la segunda mitad del siglo XX, el surgimiento del enfoque pedagógico conocido como enseñanza y aprendizaje de lenguas con fines específicos (Aguirre Beltrán, Sánchez Lobato y Santos Gargallo 2012), en el cual se basa el presente trabajo y cuyo idioma pionero fue el inglés.

La enseñanza de EFE tuvo su período de desarrollo en la década de los años 80 del pasado siglo ${ }^{7}$ y se centró principalmente en el campo de los negocios y de los servicios, así como en el académico. «La evolución de esta tendencia en la enseñanza del español ha dado lugar a la denominación de Español Profesional y Académico (EPA) y a la clasificación de dos ámbitos: Español con Fines Académicos (EFA) y Español con Fines Profesionales ${ }^{8}$ (EFP)» (Aguirre Beltrán 2012: 12). Como apuntan Aguirre, Sánchez y Santos (2012), las diferentes medidas tomadas en torno al español (implantarlo en el sistema educativo de otros países, en actividades en cooperación o comercio internacional $o$ en las industrias cinematográfica y discográfica) han producido un incremento de la demanda.

Del interés por el estudio profesional del español se desprende la necesidad de vincularlo a los aspectos socioculturales que giran en torno al idioma, pues cultura y comunicación van en una misma dirección. Como así defiende Aguirre Beltrán, «el orador debe presentar una imagen coherente con el contexto (palabras, gestos, indumentaria, símbolos) para facilitar la comunicación y fomentar la credibilidad» (2000: 40). Esta coherencia con el contexto vendrá dada si el hablante extranjero tiene nociones de los aspectos culturales que giran en torno a ciertos rituales sociales, relacionados con situaciones en las que el grado de protocolo es elevado como en intercambios comerciales o reuniones de negocios. Es decir, si la cultura ya está ligada de forma natural a la lengua, el estudiante de EFE tendrá que recibir una formación sociocultural todavía mayor, si cabe, a la de uno de EG, debido a que los contextos de uso en los que necesitará la lengua estarán mucho más convencionalizados en lo que a actitudes y a hábitos se refiere.

\section{Diseño del curso: materiales y métodos9.}

\footnotetext{
${ }^{7}$ Como asegura Grau Mena (2009), la demanda del español fue tardía en comparación con la del inglés, cuya enseñanza como lengua aplicada a ámbitos especializados se había iniciado veinte años antes.

8 EFP: el Español con Fines Profesionales consiste en la adaptación y adecuación del proceso de enseñanza-aprendizaje de la lengua a las necesidades de comunicación en la SIC (Aguirre Beltrán, 2012: 13). Este enfoque pretende «mejorar las capacidades de comprensión, expresión, interacción o mediación que se requieren para desenvolverse efectiva y eficazmente en un determinado campo de actividad profesional» (Aguirre Beltrán, 2000: 35).

${ }^{9}$ Por cuestiones de espacio, no se incluyen en este artículo los documentos mencionados. No obstante, si alguien está interesado en consultarlos (Módulos, Análisis de necesidades, Programación, Actividades) puede enviar un correo a la dirección que aparece en la página inicial de este trabajo. Para el diseño del
} 
El punto de partida es la necesidad de dar respuesta a la demanda de los estudiantes italianos para cubrir una serie de horas lectivas: se ha de diseñar - puesto que no se disponía de nada parecido con anterioridad, ni tampoco los manuales existentes reflejaban estos intereses- un plan de estudios básico de español de los negocios para adolescentes italianos. A partir de este momento, comienzan a desarrollarse las diferentes etapas metodológicas que se mencionan a continuación: en primer lugar se diseña un documento que refleja los seis bloques temáticos donde se recogen los objetivos y contenidos solicitados por la $A S L, \underline{\underline{10}}$ teniendo también en cuenta las demandas específicas del profesorado italiano -en contacto con la academia de acogida, AIP-. En una fase posterior, estos módulos se ven completados con materiales ${ }^{11}$ concretos que dan alcance a los objetivos previstos. Tras la temporalización y distribución de los materiales en diversas sesiones ( 5 o 10 sesiones de 3 o 4 horas cada una, en función de la duración de la estancia de los alumnos en la escuela), se lleva a cabo la puesta en práctica en el aula.

Para la selección de materiales y diseño de la programación se tienen especialmente en cuenta aquellas actividades que presenten destrezas integradas, que persigan un fin comunicativo y, en algunas ocasiones, se trabaja con realia, para que se enfrenten a documentos verídicos con los que tendrán que lidiar a lo largo de su vida laboral. Por su parte, para la creación de materiales propios que se incluyen también en el syllabus, se piensan actividades significativas, donde se deba usar la lengua en contextos reales, y se persigue el enfoque por proyectos, en el que se incluyen también simulaciones y presentaciones, con el fin de formar "profesionales-alumnos"12.

\subsection{Análisis de necesidades}

curso (selección de materiales) se tomaron referencias de: García Santa-Cecilia (2000), Gómez de Enterría (2009) o Cabré y Gómez de Enterría (2006)

${ }^{10}$ Los bloques temáticos en los que se divide la programación (con algunos de sus objetivos y contenidos a modo de ejemplo) son los siguientes: 1. El mundo laboral - 8h (Objetivos: Familiarizarse con las ofertas y solicitudes de empleo o Redactar un anuncio de trabajo. Contenidos: La carta de solicitud de empleo y de presentación, Los nuevos canales de búsqueda de trabajo), 2. Montamos un negocio - 12h (Objetivos: Conocer modelos de empresas españolas, Entrar en contacto con el proceso de creación de una empresa. Contenidos: Organigramas empresariales, Planes de empresa: exposiciones orales), 3. Mercadotecnia - 4h (Objetivos: Analizar anuncios en prensa, Lanzar y/o crear un producto. Contenidos: La publicidad, Las estrategias de mercadotecnia), 4. Emprendedores - 4h (Objetivos: Estudiar las bases para llegar a ser líderes y nuevos líderes. Contenidos: Trazar un plan para nuestro futuro profesional y personal), 5. Exportamos nuestra empresa - 8h (Objetivos: Conocer la economía local, Expandir las empresas. Contenidos: Negociación con extranjeros), 6. Cuestiones financieras - 4h (Objetivos: Conocer aspectos básicos sobre economía. Contenidos: La economía doméstica, Las remuneraciones y los salarios). En este grupo en concreto, por demanda del profesorado, no se trabaja el bloque 6. Como el currículo se va redefiniendo en cada ocasión, estos módulos van adaptándose a ellos.

${ }^{11}$ La recopilación de material incluye tanto la búsqueda en diferentes medios -manuales de EFE y de EG (Socios: El léxico de los negocios-Ud. 1, pp. 6-9-, Al día-B1/B2, pp. 116-117-, Practica tu españolExpresiones para hablar de trabajo, Ud. 2, pp. 14-18-, Nuevo Aula Internacional -N.A.I. 1, Ud. 4, pp. 5657; Ud. 8, p. 96; N.A.I. 3, Ud. 5, pp. 58-62-, Prisma C1 -pp. 62-63-) y páginas web (Youtube, todoele, rutaele)- como la creación propia de documentos que sirvan al alumno para alcanzar y trabajar los objetivos y contenidos seleccionados (tablas, cuestionarios, textos, Kahoot...).

12 Concepto extraído del PC EFE de Martín Peris y Sabater (2011), un plan genérico de español susceptible de ser adaptado a diferentes tipos de fines específicos. https://www.slideshare.net/negocioELE/plan-curricular-de-espaol-de-los-negocios 
Si bien es cierto que los análisis de necesidades resultan necesarios para planificar cualquier curso de lengua extranjera, lo son todavía más cuando estos tienen unas finalidades específicas. En un programa de EFE prima el uso que de la lengua harán los estudiantes $\mathrm{y}$, por ello, es fundamental tener en cuenta algunas cuestiones a partir del estudio previo de las metas que persigue cada alumno (Aguirre 2012).

Antes de llegar a la escuela, los profesores cumplimentan un test de necesidades inicial, lo que nos ayuda a seleccionar los contenidos que serán vistos en el aula; en este momento, los enseñantes escogen los módulos que prefieren trabajar en nuestra escuela, por lo que el curso queda, en cierto modo, personalizado y adaptado a cada ocasión y grupo.

En segunda instancia, serán los propios alumnos quienes, a su llegada, aporten la información necesaria para completar un segundo análisis de necesidades, más centrado esta vez en las características de los jóvenes y en sus intereses y motivaciones con respecto a la materia, así como en sus objetivos y en su relación con el idioma. Sus respuestas supusieron nuestra fuente de datos principal para establecer los objetivos concretos con respecto al diseño del curso: aumentar la motivación de los alumnos y aproximar los temas a tratar a su realidad y a su edad ${ }^{13}$, para lo cual era necesario realizar una comprobación: fue en este punto cuando se consideró necesaria la elaboración de los tres cuestionarios, que serán explicados detalladamente en $\S 4$.

\subsection{Métodos de trabajo}

Para la programación se han tenido en cuenta algunos de métodos recopilados por Aguirre Beltrán (2000:41) y por Aguirre Beltrán, Sánchez Lobato y Santos Gargallo (2012) para el diseño de un curso de $\mathrm{EFE}^{14}$ :

a) Simulaciones. En algunas actividades, los alumnos deben representar una situación real y enfrentarse a ella con los recursos lingüísticos y comunicativos que, previamente, el profesor les facilita. Este es el caso de las entrevistas de trabajo, la creación de una idea de negocio o el diseño de su propia campaña publicitaria ${ }^{15}$. Los alumnos desarrollan estas escenas reales del mundo de los negocios, lo que hace que parte de la metodología gire en torno a las simulaciones -las cuales constituyen el eje vertebrador del curso-. Como afirman los autores, este enfoque, pese a suponer una gran inversión de tiempo y una elevada carga de trabajo, proporciona a los alumnos diferentes niveles de

\footnotetext{
${ }^{13}$ El análisis de necesidades nos dejó ver que su objetivo era realizar horas lectivas (obligatorias para su currículo académico) sobre negocios en una lengua extranjera, lo cual define Grau Mena (2009) como alumnos motivados hacia la materia únicamente por sus objetivos académico-curriculares, sin ningún interés más allá de cubrir esa obligatoriedad a la que se ven abocados. Esto puede suponer un problema y dificultar el desarrollo de la clase si la falta de motivación conduce al desinterés y a la desconcentración.

${ }^{14} \mathrm{La}$ elección de estos métodos viene justificada, por una parte, por el hecho de que en un curso de negocios resulta factible realizar un proyecto general a modo de empresa, mediante el cual los estudiantes integren todos los contenidos que van trabajando. Por otra parte, las simulaciones permiten que los alumnos aprendan a desenvolverse en estos contextos reales $-\mathrm{o}$ que, al menos, recrean la realidad empresarial-y que desarrollen su estrategia comunicativa de la forma más contextualizada posible.

${ }^{15}$ Estas son algunas de las actividades que se realizan durante el curso y que permiten al alumno alcanzar el objetivo final: la creación, el desarrollo y la presentación de una idea de negocio propia.
} 
actuación entre ellos y ofrece actividades motivadoras -puesto que se pone en práctica la toma real de decisiones- y que fomentan su creatividad.

b) Proyectos. El curso, en general, está diseñado para realizar un proyecto cuyo producto final es la creación de una idea de negocio para llevarla a concurso y exponerla delante de "un grupo de inversores" (los profesores y el resto de alumnos), a modo de presentación oral en la que se incluyan todas las cuestiones trabajadas a lo largo de las dos semanas relacionadas con su idea de negocio. El proyecto es, por tanto, el punto de partida y llegada del programa.

\section{Una sesión a modo de ejemplo. Día 6: Las entrevistas de trabajo}

La temática del sexto día es Las entrevistas de trabajo. Para aproximarnos a este tema, los alumnos realizarán diversas actividades: el visionado de vídeos que servirán como modelo de imitación, la lectura de textos que proporcionará input léxico y estructural a los estudiantes y la puesta en práctica de simulaciones -entrevistas reales, creadas y recreadas por ellos- les permitirán entrar en contacto con este género, no solo con sus especificidades en la cultura hispana sino también en otras culturas.

Como se puede apreciar en la Tabla 1, las sesiones de cuatro horas están divididas en tres bloques. A continuación, se muestra un resumen de las tareas realizadas en este día, así como se detallan las destrezas ${ }^{16}$, el tipo de agrupamiento y los contenidos de cada una de ellas. El hecho de seleccionar este día encuentra su justificación en los resultados de los cuestionarios ( $(5)$, pues es una de las actividades preferidas de los alumnos. Además, es una de las que más repercusión tiene en su proyecto final.

\begin{tabular}{|c|c|c|}
\hline & \multicolumn{2}{|c|}{ SESIÓN 6: LAS ENTREVISTAS DE TRABAJO } \\
\hline \multirow{9}{*}{$\begin{array}{l}\text { Clase } \\
1(1 \mathrm{~h} \\
10 \\
\min )\end{array}$} & \multirow{3}{*}{$\begin{array}{c}\text { 1. Lluvia de ideas: ¿Qué es } \\
\text { una entrevista? }\end{array}$} & Destrezas: EO \\
\hline & & Agrupamiento: clase plenaria \\
\hline & & Contenidos: léxicos (vocabulario de las entrevistas). \\
\hline & \multirow{2}{*}{$\begin{array}{l}\text { 2. Vídeo: Errores más } \\
\text { frecuentes en las entrevistas de } \\
\text { trabajo (y posterior puesta en } \\
\text { común) }\end{array}$} & Destrezas: $\mathrm{CO}, \mathrm{EO}$ \\
\hline & & Agrupamiento: clase plenaria \\
\hline & $\begin{array}{l}\text { trabajo (y posterior puesta en } \\
\text { común) } \\
{[\text { https://www.youtube.com/watc }} \\
\quad h ? v=\text { oMTOfoVKnTg] }\end{array}$ & $\begin{array}{l}\text { Contenidos: léxicos (temas de una entrevista), } \\
\text { socioculturales (qué no se debe hacer en una entrevista en } \\
\text { España), funcional (responder adecuadamente a las } \\
\text { preguntas del entrevistador). }\end{array}$ \\
\hline & & Destrezas: $\mathrm{CE}$ \\
\hline & 3. Texto: Las culturas y las & Agrupamiento: individual \\
\hline & $\begin{array}{l}\text { entrevistas de trabajo. } \\
\text { [Manual Socios, pp. 142-143] }\end{array}$ & $\begin{array}{l}\text { Contenidos: socioculturales (cómo evitar malentendidos } \\
\text { culturales en contextos laborales, saber actuar y } \\
\text { relacionarse en otras culturas). }\end{array}$ \\
\hline & & Destrezas: $\mathrm{CE}, \mathrm{IO}$ \\
\hline & 4. Ficha 8. Preguntas & Agrupamiento: gran grupo y parejas \\
\hline $\begin{array}{l}2(1 \mathrm{~h} \\
15 \mathrm{mi} \\
\mathrm{n})\end{array}$ & $\begin{array}{c}\text { en las entrevistas (puesta en } \\
\text { común y dramatización) } \\
\text { [Creación propia] }\end{array}$ & $\begin{array}{l}\text { Contenidos: socioculturales (qué es frecuente preguntar } \\
\text { en una entrevista de trabajo según la empresa), funcionales } \\
\text { (saber comunicar una opinión, saber responder a una } \\
\text { pregunta formal), léxico (fórmulas de tratamiento y }\end{array}$ \\
\hline
\end{tabular}

${ }^{16}$ Las diferentes destrezas trabajadas son: expresión oral (EO), expresión escrita (EE), interacción oral (IO), comprensión oral (CO) y comprensión escrita (CE). 


\begin{tabular}{|c|c|c|}
\hline & & estructuras del registro formal). \\
\hline & & Destrezas: EE, IO \\
\hline & 5. Creación d & Agrupamiento: grupos del proyecto (3-4) \\
\hline & $\begin{array}{l}\text { para un puesto concreto } \\
\text { [Creación alumnos] }\end{array}$ & $\begin{array}{l}\text { Contenidos: funcionales (formular preguntas para pedir } \\
\text { información, expresar opinión, etc.), socioculturales } \\
\text { (adecuación a un puesto de trabajo concreto). }\end{array}$ \\
\hline & & Destrezas: IO \\
\hline & en prácti & Agrupamiento: grupos del proyecto (3-4) \\
\hline $\begin{array}{l}\text { Clase } \\
3(1 \mathrm{~h} \\
5 \mathrm{~min})\end{array}$ & $\begin{array}{l}\text { realización de las entrevistas } \\
\text { de trabajo } \\
\text { [Creación alumnos }]\end{array}$ & $\begin{array}{l}\text { Contenidos: Los grupos (empresas) deben entrevistar a } \\
\text { los compañeros y ser entrevistados posteriormente por } \\
\text { otras empresas (otros grupos de alumnos), de forma que } \\
\text { ponen en práctica todo lo trabajado a lo largo de la sesión. }\end{array}$ \\
\hline
\end{tabular}

Tabla 1. Sesión 6: Las entrevistas de trabajo

\section{Los cuestionarios: (auto)evaluar el curso y el aprendizaje.}

Esta etapa constituye el núcleo del estudio ante el que nos encontramos: el diseño de unos cuestionarios que recojan la totalidad de conceptos trabajados a lo largo del curso, así como las principales habilidades que se espera que los alumnos hayan desarrollado y las opiniones relacionadas con la calidad del curso. Dicha fase incluye también la realización de las pruebas por parte de los 28 alumnos de nuestro grupo meta. Los pasos seguidos para su preparación son los siguientes:

a) Diseño: en base a la estructura del curso, se elabora un test ${ }^{17}$ en el que se tienen en cuenta todos los aspectos trabajados:

\begin{tabular}{||l|l||}
\hline Autoevaluación de: & Evaluación del curso \\
\hline \hline $\begin{array}{l}\text { Habilidades: } 8 \text { preguntas sobre lo que saben o } \\
\text { son capaces de hacer }{ }^{18} \text {, que se ha practicado en }\end{array}$ & $\begin{array}{l}\text { Se incluyen } 5 \text { preguntas sobre: } \\
\text { el interés personal de cada estudiante en el } \\
\text { mundo de los negocios. }\end{array}$ \\
$\begin{array}{l}\text { hacer un análisis DAFO }{ }^{19} \text { o elaborar una oferta } \\
\text { de empleo). }\end{array}$ & $\begin{array}{l}\text { la influencia que puede tener este curso sobre su } \\
\text { futuro laboral. } \\
\text { - las actividades que les han resultado más o } \\
\text { menos útiles, para obtener así información. }\end{array}$ \\
$\begin{array}{ll}\text { la parte teórica del curso (i.e. Modelos de } \\
\text { empresas españolas, } \\
\text { mercadotecnia o expresiones coloquiales para } \\
\text { hablar de trabajo). }\end{array}$ & $\begin{array}{l}\text { (i.e. Interés hacia el tema, utilidad de los } \\
\text { contenidos o actividades que más/menos han } \\
\text { gustado). }\end{array}$ \\
\hline
\end{tabular}

${ }^{17}$ El cuestionario, diseñado en base a una escala Likert de cinco puntos, consta de 21 preguntas en total: 19 de respuesta cerrada, donde han de evaluar sobre 5 (caritas más o menos sonrientes) su conformidad con la afirmación (siendo 5 "totalmente de acuerdo" y 1 "totalmente en desacuerdo"), y 2 de respuesta abierta. El modelo puede solicitarse a la dirección de correo electrónico referida en la primera página, así como el resto de gráficos (resultados individualizados para cada pregunta) que no se incluyen en $\$ 5.1$. Los gráficos corresponden al cómputo de las respuestas de los alumnos.

${ }^{18}$ Los saberes o conocimientos que un estudiante de lengua extranjera ha de poseer según el PCIC se recogen en nuestro cuestionario, pues los alumnos deben tomar conciencia sobre lo que saben, saben hacer, saben aprender y saben ser (https://cvc.cervantes.es/ensenanza/biblioteca ele/marco/cap 05.htm).

${ }^{19} \mathrm{El}$ análisis DAFO, también conocido como análisis FODA o DOFA, es una herramienta de estudio de la situación de una empresa, institución, proyecto o persona, analizando sus características internas y su situación externa en una matriz cuadrada. Las siglas representan los conceptos que se analizan en dicho análisis: debilidades, amenazas, fortalezas y oportunidades. 
Tabla 2. Diseño de los cuestionarios

b) Realización: El último día de clase, los 28 alumnos reciben el documento y, tras una breve explicación sobre su funcionamiento, lo completan de manera individual y anónima, para alcanzar el mayor grado de sinceridad posible.

c) Recogida y recopilación de datos: las respuestas obtenidas se suman para, posteriormente, obtener los resultados plasmados en los gráficos, algunos de los cuales se pueden ver en $\$ 5.1$.

La finalidad que se persigue en esta fase tiene dos vertientes. Por una parte, comprobar si el curso resulta motivador -el análisis inicial mostraba la falta de interés hacia la temática- y adecuado, pues se trata de un programa nuevo. Esto lo podemos apreciar en el Cuestionario 3, Evaluación del curso. Además, nos permite conocer cuáles son las actividades que tienen más éxito o el tipo de tareas que los motiva más. Por otra, como el profesor no evalúa en sentido estricto -pues se trata de un curso breve y no se realiza examen-, la autoevaluación les sirve para tomar conciencia de su proceso de aprendizaje, así como de los conocimientos que han adquirido y de las habilidades que son capaces de desarrollar en español en relación con el mundo de los negocios. De tal manera, comprobamos si nuestro curso cumple los objetivos de los alumnos y si es útil también en relación con las pautas de la ley $A S L$.

\subsection{Resultados}

En primer lugar, atendiendo al cuestionario de Autoevaluación de Habilidades, se observan los siguientes datos: un 47,3\% recibe la calificación de 5: casi la mitad de la clase afirma ser capaz de realizar con éxito las tareas descritas. Por otra parte, se obtiene un $49,1 \%$ para la calificación de 4 , lo que significa que, aunque no a la perfección, casi la otra mitad las ha adquirido ampliamente. El 3,6\% restante corresponde a las calificaciones para 3: saber realizar la tarea de forma sencilla, sin mucha soltura.

Para el segundo -el correspondiente a la Autoevaluación de los Conocimientos adquiridos durante el curso- se aprecia que la nota de 5 (adquisición total de conocimiento) obtiene un $41,7 \%$, mientras que un $53,5 \%$ corresponde a 4 (han adquirido un conocimiento bastante significativo) y únicamente un $4,8 \%$ no se sienten seguros con respecto a los nuevos conceptos (calificación de 3: sé lo básico).

Por último, los resultados son también muy positivos para el tercero, puesto que uno de nuestros principales objetivos era, precisamente, tratar de configurar un programa didáctico que conectara con el grupo meta y creara cierta afinidad entre la temática y el alumnado. Y eso parece demostrar esta última parte de la encuesta, la correspondiente a la Evaluación del curso: un 56,4\% de las respuestas corresponde a la calificación de 5 (satisfacción e interés pleno por el curso), un 38,6\% para el 4 (contentos aunque no les genera un gran entusiasmo) $\mathrm{y}$, por último, el $5 \%$ restante correspondería a las calificaciones que recibió la puntuación de 3 , ni contentos ni descontentos con el programa y con un sentimiento neutro hacia el tema. 


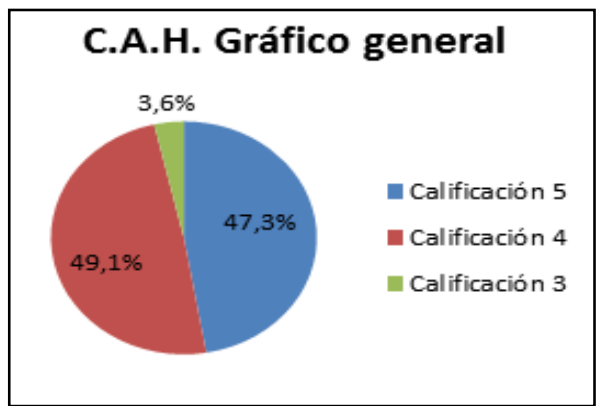

Gráfico 1. Resultados Cuestionario Autoevaluación Habilidades

\section{C.E.C. Gráfico general}

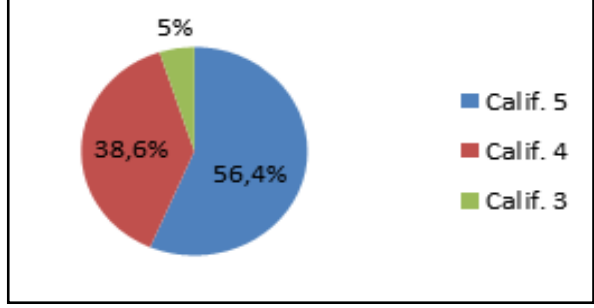

Gráfico 3. Resultados Cuestionario Evaluación Curso

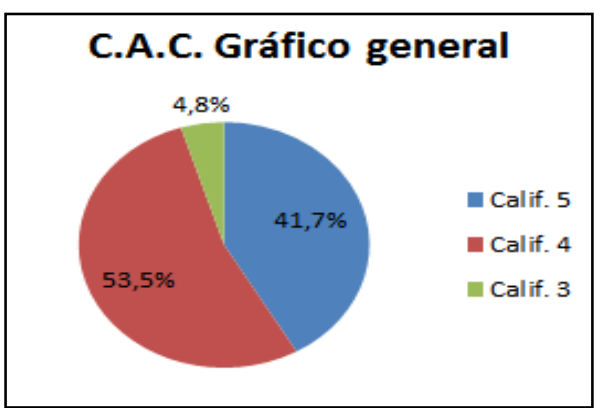

Gráfico 2. Resultados Cuestionario Autoevaluación Conocimientos

\section{Conclusiones}

El diseño de un curso de EFE dirigido a adolescentes permite ampliar el caudal de materiales del que disponíamos anteriormente. No obstante, no se presenta aquí la totalidad de sesiones desarrolladas puesto que, por una parte, corresponde esto a otra comunicación que está en curso y, por otra, se persigue un fin distinto: mostrar la tendencia mayoritaria de las respuestas que los estudiantes dieron a los cuestionarios. Esto funciona como herramienta de validación de la programación en lo que respecta a su significatividad, utilidad y capacidad motivadora. Las dinámicas planteadas en el aula han dado como resultado una propuesta didáctica que combina la teoría y la práctica, a la par que pretende alcanzar grados de significación y comunicatividad elevados. Asimismo, los objetivos se han visto confirmados gracias a la realización de los cuestionarios planteados.

Los datos expuestos en $\S 4$-donde se aprecia una amplia mayoría de respuestas que oscilan entre 4 y 5 - demuestran que se ha alcanzado, al menos de forma parcial, el desarrollo de una experiencia práctica que cubre las necesidades de un grupo de alumnos adolescentes y que refleja las demandas requeridas por $A S L$. También se prueba que estos materiales resultan útiles para los estudiantes y logran que ellos perciban la relación entre metas, contenidos y actividades, pues así se ve en sus contestaciones (cuestionario de Evaluación del curso, tanto en las preguntas 1-5 como en las preguntas 6 y 7, de respuesta abierta). El cambio con respecto a su motivación se erige como una de las conclusiones más satisfactorias: mientras que en el análisis de 
necesidades del primer día expresaban su desinterés hacia la materia, los resultados revelan un sentimiento positivo hacia el tema de los negocios, alcanzando así la finalidad primordial. Las actividades prácticas y de implicación creativo-emocional, así como las dinámicas llevadas a cabo son, seguramente, la causa de este crecimiento, ya que son las mejor valoradas por parte de los estudiantes.

De tal manera, queda así formalizado un curso de EFE para adolescentes, respaldado por las opiniones y perspectivas de los propios alumnos que, al fin y al cabo, son los que constituyen el foco de cualquier proceso de aprendizaje y quienes verifican la validez y el éxito de una programación.

\section{Bibliografía}

AgUiRRE Beltrán, B. (2000). «El español para la comunicación profesional. Enfoque y orientaciones didácticas». En Actas del I Congreso Internacional de Español con Fines Especificos. Recuperado el 03/04/2018, de https://cvc.cervantes.es/ensenanza/biblioteca_ele/ciefe/pdf/01/cvc_ciefe_01_0006.pdf

AGUiRRE BELTRÁN, B. (2012). «Enseñanza y aprendizaje de lenguas extranjeras con fines específicos», en Aprendizaje y enseñanza de español con fines especificos. Comunicación en ámbitos académicos y profesionales. Madrid: SGEL.

Aguirre Beltrán, B., J. SÁnchez lobato y I. SAntos Gargallo (2012). La enseñanza/aprendizaje del español para fines específicos: Marco general. Madrid: Universidad Complutense de Madrid.

CABré, M. T. y J. GÓMEZ DE EnTERRía (2006). La enseñanza de los lenguajes de especialidad. La simulación global. Madrid: Gredos.

Grau Mena, I. (2009). El español con fines específicos [en línea]. Director: Dr. Robert Tanzmeister. Tesis doctoral. University of Vienna.

Instituto Cervantes (2006): Plan curricular del Instituto Cervantes. Niveles de referencia para el español. Madrid: Instituto Cervantes - Biblioteca nueva.

Martín Peris, E. y M.L. SABAter Fernández (2011). Plan curricular de Español de los negocios de la Fundación Comillas. Comillas: CIESE. 